\title{
Review Article \\ Exercise Interventions in Children with Cancer: A Review
}

\author{
Tseng-Tien Huang and Kirsten K. Ness \\ Department of Epidemiology and Cancer Control, St. Jude Children's Research Hospital, Memphis, TN 38105, USA \\ Correspondence should be addressed to Kirsten K. Ness, kiri.ness@stjude.org
}

Received 8 July 2011; Revised 3 September 2011; Accepted 3 September 2011

Academic Editor: Julie Blatt

Copyright ( $\odot 2011$ T.-T. Huang and K. K. Ness. This is an open access article distributed under the Creative Commons Attribution License, which permits unrestricted use, distribution, and reproduction in any medium, provided the original work is properly cited.

\begin{abstract}
The purpose of this review is to summarize literature that describes the impact of exercise on health and physical function among children during and after treatment for cancer. Relevant studies were identified by entering the following search terms into Pubmed: aerobic training; resistance training; stretching; pediatric; children; AND cancer. Reference lists in retrieved manuscripts were also reviewed to identify additional trials. We include fifteen intervention trials published between 1993 and 2011 that included children younger than age 21 years with cancer diagnoses. Nine included children with an acute lymphoblastic leukemia (ALL) diagnosis, and six children with mixed cancer diagnoses. Generally, interventions tested were either in-hospital supervised exercise training or home based programs designed to promote physical activity. Early evidence from small studies indicates that the effects of exercise include increased cardiopulmonary fitness, improved muscle strength and flexibility, reduced fatigue and improved physical function. Generalizations to the entire childhood cancer and childhood cancer survivor populations are difficult as most of the work has been done in children during treatment for and among survivors of ALL. Additional randomized studies are needed to confirm these benefits in larger populations of children with ALL, and in populations with cancer diagnoses other than ALL.
\end{abstract}

\section{Introduction}

Progress in treatments for childhood cancer have greatly improved cure rates, with 5-year survival now approaching $80 \%$ [1]. This has resulted in a growing population of childhood cancer survivors. In 2006, there were more than 11 million cancer survivors in the United States; three times the number of survivors in 1971 [2]. It is estimated that 1 in 810 individuals under the age of 20 is a survivor of childhood cancer and that 1 in 640 individuals between the ages of 20 and 39 years has successfully survived childhood cancer [3]. Improving survival rates, however, does not come without consequences. Treatment of childhood cancer is associated with a spectrum of late effects, including impaired growth and development, cognitive dysfunction, diminished neurological function, cardiopulmonary compromise, musculoskeletal sequelae, and secondary malignancy [4-6]. Oeffinger et al. [6] reported that one-third of childhood cancer survivors have severe or life-threatening medical complications 30 years after diagnosis. Therefore, attention today is focused not only on survival but also on the quality of survival.

Impaired physical fitness has been reported during and after childhood cancer treatment [7-12]. Impaired physical fitness typically includes reduced cardiopulmonary function, decreased muscle strength, fatigue, and altered physical function. Treatments for childhood cancer, including radiotherapy, chemotherapy, and surgery, can result in acute and long-term injury to the heart, lungs, and skeletal muscles, systems necessary for optimal physical fitness $[8$, 13-19]. Additionally, reduced levels of physical activity both during and after treatment for childhood cancer can contribute to cardiac deconditioning and skeletal muscle atrophy, ultimately limiting opportunities for participation in recreational activities and life roles that are dependent on adequate physical fitness. Exercise intervention has the potential to improve cardiopulmonary and musculoskeletal function, perhaps preventing long-term deficits in physical fitness if incorporated during or soon after treatment in children with cancer diagnoses [20-24]. 
Another factor that may be associated with impaired physical fitness among childhood cancer survivors is cancerrelated fatigue. Fatigue during and after treatment has the potential to have a negative impact on physical activity and on psychosocial well-being. A recent study reported that the prevalence of cancer-related fatigue was over three times higher in long-term survivors of childhood cancer when compared to the general population (OR: 3.29; 95\% CI: 1.9-5.70) [25]. In another investigation that evaluated associations between demographic and medical factors and health-related quality of life (HRQOL) among pediatric cancer survivors, Meeske et al. [26] found that fatigue was the most powerful predictor of functional status and HRQOL. Given that there is evidence that exercise and physical activity programs can reduce fatigue, and enhance psychosocial health in survivors of adult cancer, such interventions may have a similar effect in the pediatric population [27].

This review of the literature indicates that there is growing evidence for the positive effects of physical training on organ system function, fatigue and physical well-being in children during and after treatment for cancer [20, 28]. However, the optimal intervention modality and the intensity, timing, and duration of the intervention are difficult to determine. In the published literature, very few exercise interventions undertaken in the pediatric cancer or pediatric cancer survivor populations have been randomized clinical trials, and, across studies, the components of aerobic training, resistance, and flexibility exercises are implemented with differing intensity, timing, and duration. In addition, the sample sizes are small, limited primarily to study populations with acute leukemia diagnoses, and include widely varied outcome measures, making it difficult to draw firm conclusions or compare results between trials. We summarize here the literature that describes the effects of exercise intervention on immune system function, cardiopulmonary health, skeletal muscle strength, fatigue, and overall physical well-being among children during and after treatment for cancer.

\section{Methods and Search Results}

This paper summarizes exercise intervention studies among children with cancer and is limited to studies that tested or described exercise intervention in children diagnosed with a primary pediatric cancer when younger than 21 years of age, and, includes only manuscripts available as fulltext in the English language. Studies were identified by searching the PUBMED database with the terms exercise; aerobic training; resistance training; stretching; pediatric; children; cancer. Reference lists of retrieved studies were also assessed to identify additional trials. The search of the Pubmed database initially resulted in a total of 48 citations. Of these, we excluded 31 citations (3 review only, 5 not available in English, 17 no exercise intervention, and 6 adultcancer survivors only). We include 17 published manuscripts documenting 15 studies published by June of 2011. A review of the reference lists from the retrieved manuscripts did not identify any additional papers. When reporting the outcomes of each study, if numerical results were available, effect sizes were converted to Cohen's $d$, representing standard deviations of change or differences in standard deviations between groups [29], to allow for easier comparison of the magnitude of the exercise intervention responses among studies.

2.1. Exercise Intervention Studies among Pediatric Survivors. A summary of the 15 published studies included in this review examining exercise intervention for children with cancer is shown in Tables 1 and 2. A total of 302 children with cancer, survivors of childhood cancer, or normal controls participated in the 15 trials; 46 were young adults [30] and 256 were children or adolescents [21-23, 3143]. Of these 15 exercise interventions during or after pediatric cancer treatment, seven included a control group or control intervention, nine employed supervised training with aerobic, resistance, and/or flexibility training with or without home-based exercises [21-23, 31-40, 43], five tested enhanced physical activity (EPA) interventions [30, 34, 36, $39,42]$, and one used an individualized home-based exercise program [41]. We differentiate between nonrandomized (Table 1) and randomized trials (Table 2) to highlight the need for additional experimental evidence to evaluate the effects of exercise intervention for children with cancer. Only 121 children with cancer diagnoses have participated in four randomized controlled trials; 41 were children and adolescents with ALL during maintenance chemotherapy $[21,36]$ and 70 were the survivors of childhood cancer with mixed diagnoses $[33,42]$. We also differentiate between exercise and EPA by noting that exercise implies a specific training regimen with established frequency, intensity, and duration and that EPA includes dynamic activities completed during the performance of everyday tasks [44]. The majority of the interventions included only patients with acute lymphoblastic leukemia (ALL) diagnoses; only six studies were done in study populations with mixed diagnoses [30, $31,34,38,39,42]$. One study was completed in children with ALL during the first six months of medical treatment [32], and six were completed among children with ALL during the maintenance or continuation phase of medical treatment [21, 23, 35-37, 43]. Outcome measures described included (1) immunological function $[31,35]$, (2) cardiovascular fitness $[21-23,35,36,38,40]$, (3) muscle strength and flexibility $[21-23,33,34,36,40,43]$, (4) fatigue or sleep efficacy [30, 34, 40-42], (5) general physical function [9, 21, $32,33,36,40]$, and (6) quality of life $[9,21,32,34,39]$.

\subsection{Effect of Exercise on Immune Suppression and Growth} Factors. Chemotherapy treatment for pediatric cancer suppresses the immune system and may interfere with normal growth, increasing susceptibility to infection and stunting or delaying musculoskeletal development during treatment [45-47]. Concern about the effects of exercise on immune function and growth factors includes the possibility that exercise may tax an already compromised immune or endocrine system and either delay recovery or further impact normal skeletal growth [48]. A pilot study by Ladha et al. 


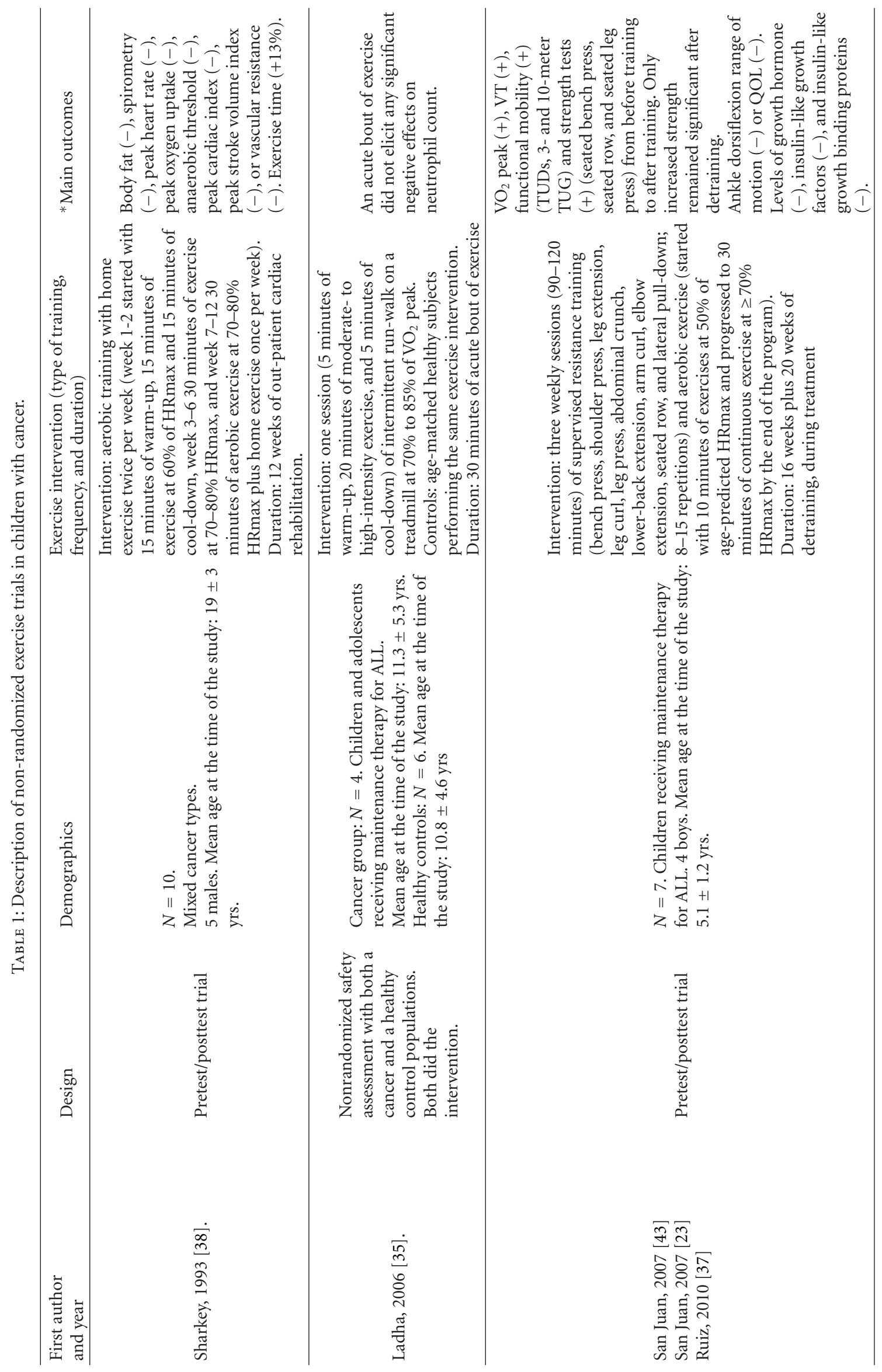




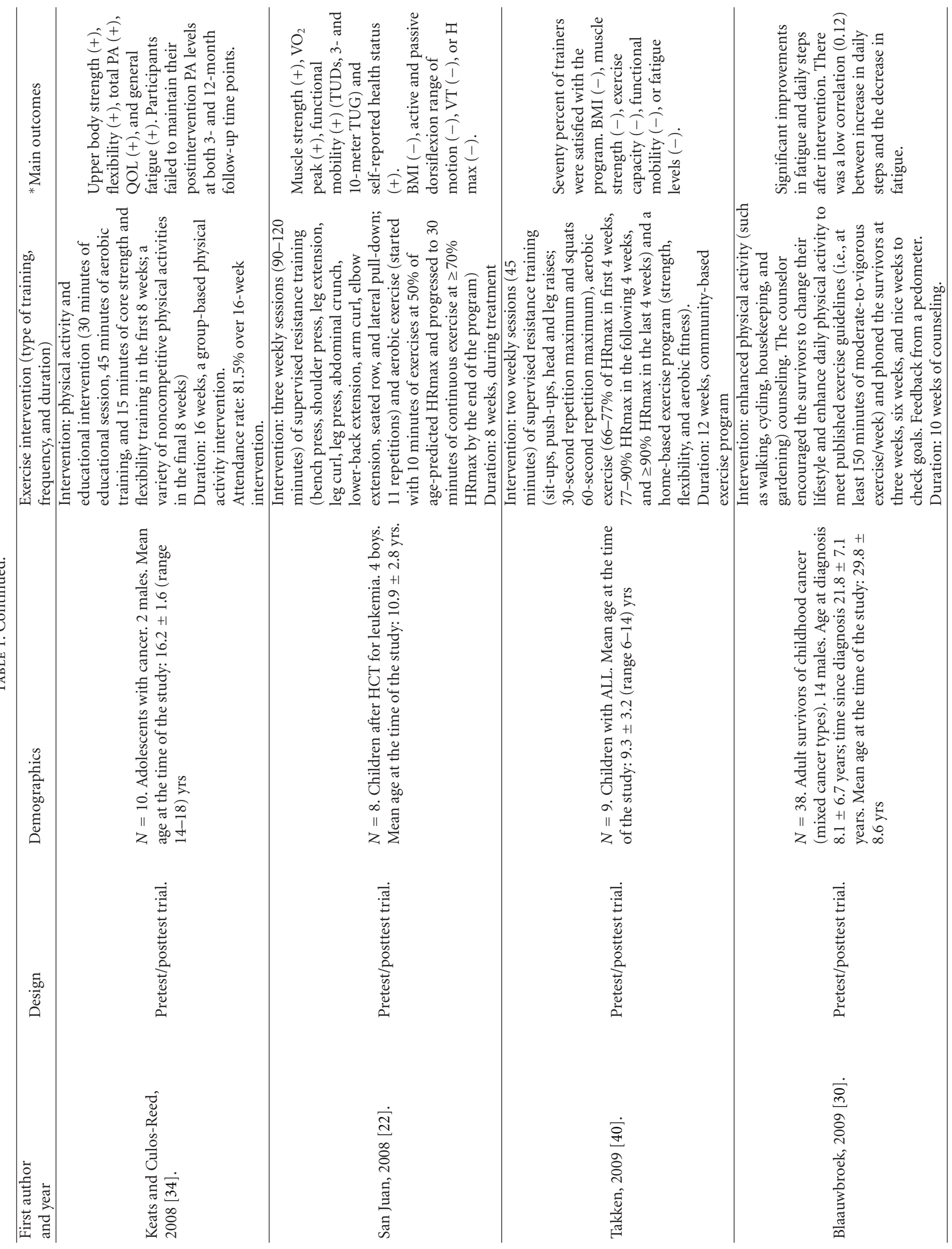




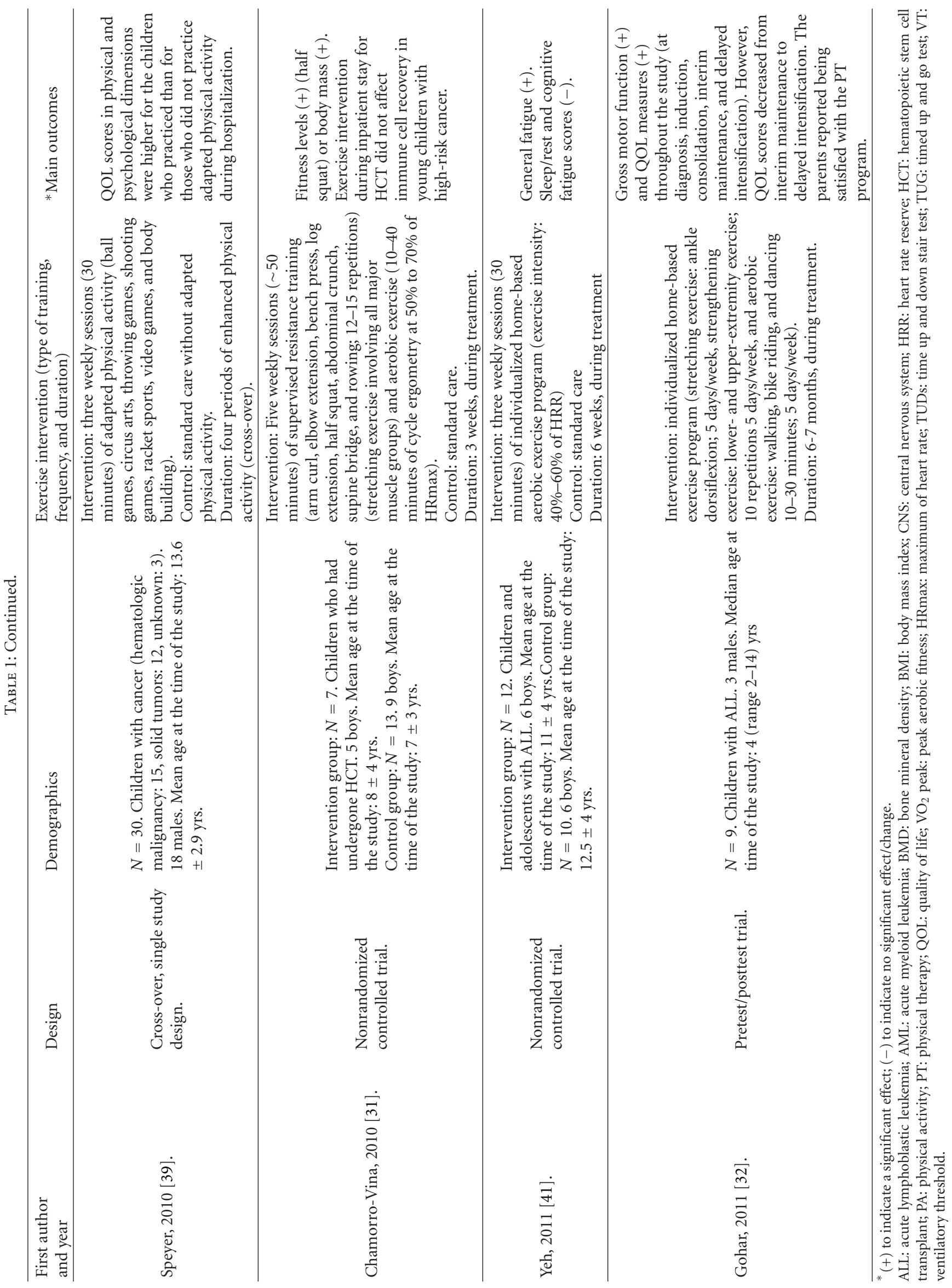




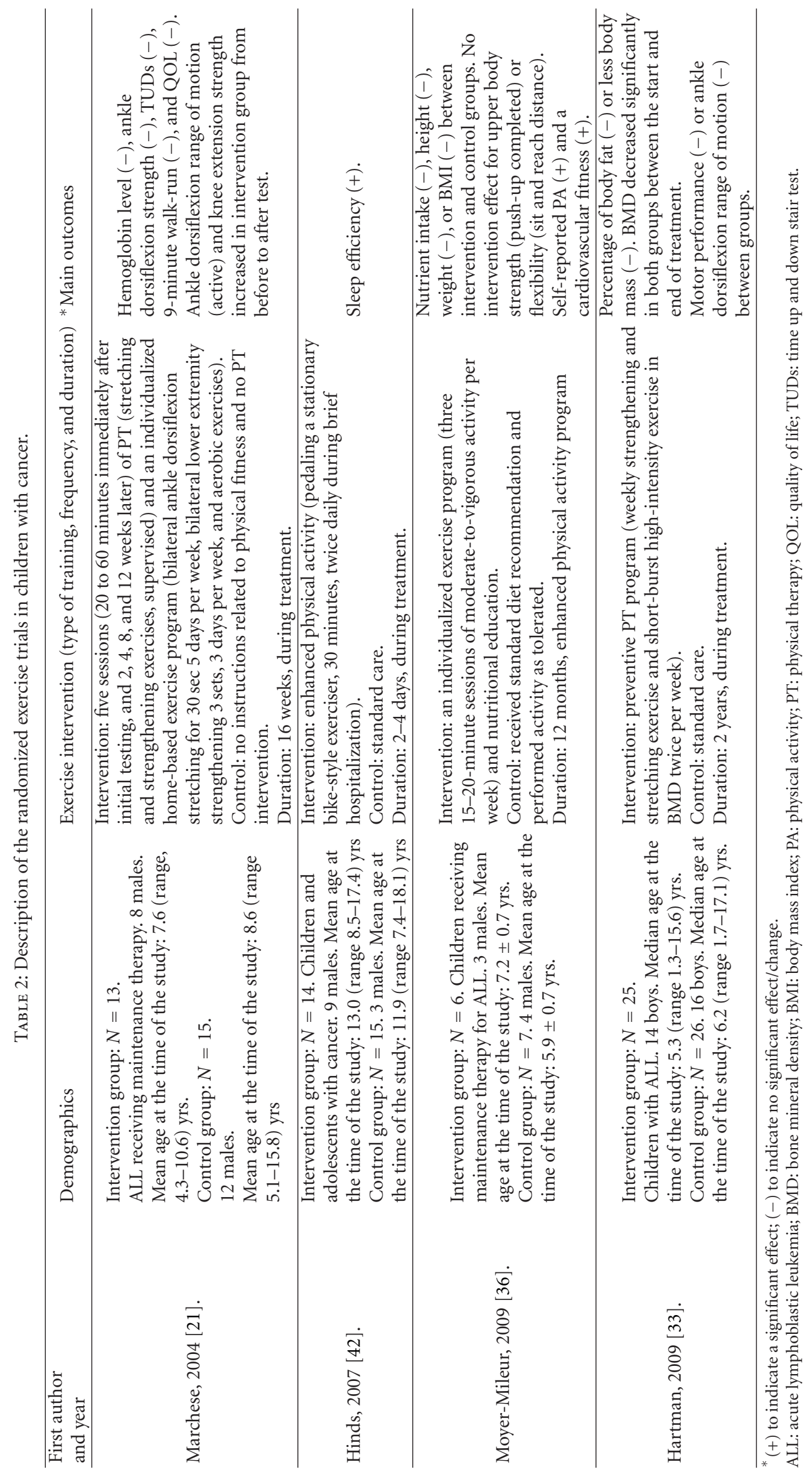


[35] investigated the effects of an acute bout (30 minutes) of exercise (heart rate $70-85 \%$ of peak oxygen uptake capacity $\left(\mathrm{VO}_{2}\right.$ peak $\left.)\right)$ on neutrophil counts and immune function in children undergoing maintenance therapy for ALL ( $n=$ 4 ; mean age $11.3 \pm 5.3$ years). They found no deleterious effects of this intervention on immune function. Their work is supported by Chamorro-Vina et al. who demonstrated that a daily 3-week in-hospital moderate intensity exercise training regimen, including both supervised resistance and aerobic components, did not affect immune cell recovery in 7 children who had undergone hematopoietic stem cell transplantation [31]. This same group of authors also report no significant impact of a 3 times per week 16week aerobic and resistance training intervention on levels of growth hormone, insulin-like growth factors, and insulinlike growth binding proteins levels (IGFBP-2 and -3) [37] in children with ALL. In this study, protein levels of IGFBPs remained stable even after 20 weeks of detraining.

2.3. Effect of Exercise on the Cardiopulmonary System. Cardiopulmonary fitness is impaired in children during treatment and among survivors of childhood cancer $[9,10]$. Reports of the effects of exercise intervention on the cardiopulmonary system during treatment are mixed. Marchese et al. [21] examined effects of exercise on cardiovascular response in children (aged 4-15 years) receiving maintenance therapy for ALL. Participants were randomly assigned into a physical therapy (PT) intervention group with home-based aerobic training two times per week $(n=13)$ or a control (nonPT intervention) group $(n=15)$. Following a four-month intervention, these authors reported no cardiopulmonary response to training as assessed by a nine minute runwalk test. Additionally, more than $50 \%$ of the children scored below the 25th percentile for cardiopulmonary fitness when compared to the normative sample in the American Alliance of Health, Physical Education, Recreation and Dance Association Guidelines at both the pre- and posttest assessments. Similarly, Takken et al. [40] implemented a 12 -week community-based exercise program in 9 children with ALL (aged 6-14 years) and found no cardiopulmonary response to training as assessed by standardized cardiopulmonary exercise testing. Of note, some children in this study complained that the training program was boring, too intense, and hard to combine with their other activities which may have limited compliance. Moyer-Mileur et al. [36], who provided a home-based intervention to children with ALL during the maintenance phase of chemotherapy, report slightly more promising results. In a much longer duration intervention, with a perhaps more palatable intensity of training, they assigned thirteen 4-10-year-old children to either 15-20 minutes of moderate to vigorous physical activity 3 times per week $(n=6)$ or to no intervention $(n=7)$. After 12 months, regular physical activity and cardiopulmonary fitness were assessed with a pedometer and a Progressive Aerobic Cardiovascular Endurance Run test (PACER) [49], respectively. The authors reported that the exercise group recorded more steps on the pedometer $(P=$ 0.06 , Cohen's $d=1.12$ ) and performed slightly better on the
PACER $(P=0.05$, Cohen's $d=1.22)$ than the control group at the end of the intervention.

It appears that hospital type supervised exercise interventions have better cardiopulmonary outcomes than do those that are home or community based. San Juan et al. [9] reported positive results after implementation of a 16-week supervised (in-person) exercise program among 7 children with ALL, 4-7 years of age, also during the maintenance phase of chemotherapy. Their study population achieved a significant increase in both ventilatory threshold (before training $15.8 \pm 3.3$; after training $20.7 \pm 2.9$ milliliters per kilogram per minute $(\mathrm{mL} / \mathrm{kg} / \mathrm{min}), P<0.05$, Cohen's $d=$ 1.58 ) and peak oxygen uptake (before training $24.3 \pm 5.9$; after training $30.2 \pm 6.2 \mathrm{~mL} / \mathrm{kg} / \mathrm{min}, P<0.05$, Cohen's $d=0.97$ ). A subsequent study by San Juan et al. [9] showed similar benefits for an 8-week supervised exercise training program among 8 children after HCT.

Supervised exercise training also appears to have promise for childhood cancer survivors with long-term cardiopulmonary compromise. A study by Sharkey et al. [38] examined the effects of a 12-week aerobic training program among childhood cancer survivors who had been treated with anthracycline chemotherapy (cumulative dosage $349 \pm$ 69 milligrams per meter squared $\left(\mathrm{mg} / \mathrm{m}^{2}\right)$ ). Among the 10 patients who completed the twice weekly program (mean age $19 \pm 3$ years; mean time since diagnosis $8 \pm 4$ years), there was an average increase in exercise time on cardiopulmonary exercise testing (CPET) of 13 percent (\%) from before to after test $(P<0.05$, Cohen's $d=0.47)$. They also reported a trend toward improvement in peak oxygen uptake $(P=$ NS, Cohen's $d=0.36)$ and anaerobic threshold $(P=\mathrm{NS}$, Cohen's $d=0.58$ ), but no significant changes in body fat, spirometer parameters, cardiac index, or stroke volume index. Unfortunately, although these 10 participants showed some improvement in exercise tolerance, their exercise capacity remained substantially lower than those of normal subjects.

2.4. Effect of Exercise on the Musculoskeletal System. Cancer therapy in children also impacts the musculoskeletal system. Limited range of motion, loss of muscle mass, and reduced muscle strength are common among children with cancer and among survivors [33, 50-52]. Fortunately, the early intervention research targeting these impairments is very promising. Improved muscle strength and flexibility is reported following training among children during maintenance therapy for ALL $[9,21]$ and in groups of children with mixed cancer diagnoses [34]. In their 12-week home-based PT intervention for children during maintenance therapy for ALL ( $n=13$ intervention group, $n=15$ control group), Marchese et al. [21] reported that stretching and resistance training improved ankle range of motion $(P<0.01$, Cohen's $d=0.62)$ and knee extension strength $(P<0.01$, Cohen's $d=0.35$ ). In another intervention during ALL maintenance therapy among 7 children 4-7 years of age, San Juan et al. [9] implemented resistance training for the major muscle groups and reported muscle strength gains $(P<0.05$, Cohen's $d=$ 0.85 to 1.48) after just eight weeks of training [9]. These gains 
were maintained after a 20-week detraining period [9]. In a longer intervention program (2 years) designed to prevent bone loss in children during treatment for ALL (intervention group $n=25$, control group $n=26$ ) Hartman et al. [33] reported that stretching and twice daily short-burst highintensity exercise resulted in improved dorsiflexion range of motion $(P=0.001$, Cohen's $d=0.94)$, but not in improved bone health. In a group-based physical activity intervention with a group of adolescent cancer survivors with mixed diagnoses $(n=10)$, Keats and Culos-Reed [34] reported improved upper body strength $(P<0.05$, Cohen's $d=0.64)$ after 16 weeks of participation.

2.5. Effect of Exercise on Fatigue. Fatigue is a common symptom in children during and following cancer treatment $[53,54]$. Both exercise and EPA type interventions show some efficacy in the management of fatigue during and after cancer chemotherapy in children [30, 34]. However, it appears that fatigue reduction also requires a training response. In three studies, where the response to training was positive, fatigue reduction was evident and even persisted, whereas in one study, where the exercise response was null, so was the fatigue reduction response. Yeh et al. [41] reported reduced levels of fatigue $(P=0.03$, Cohen's $d=0.54)$ among children with ALL $(n=12)$ who completed a sixweek home-based aerobic exercise program when compared to a control group who did not $(n=10)$, and Blaauwbroek et al. [30] reported reduced levels of fatigue $(P<0.005$, Cohen's $d=-0.92$ ) and increased levels of physical activity $(P<0.005$, Cohen's $d=0.94)$ after 10 weeks of a homebased physical activity counseling intervention in childhood cancer survivors. The Blaauwbroek study was implemented in survivors ( $n=38$ ) who were on average 30 years of age and 22 years from their original cancer diagnosis. Importantly, fatigue reduction was maintained in their study population at a three-year follow-up time point. The results of a study by Keats and Culos-Reed [34] also demonstrated a reduction in fatigue $(P=0.01$, Cohen's $d=0.69)$ after a 16-week group-based physical activity intervention in survivors of pediatric cancer $(n=10)$. In contrast, a 12 -week community-based exercise training program where there was no exercise response, perhaps because of noncompliance, also demonstrated no fatigue reduction response ( $P=$ NS, Cohen's $d=-0.26$ ) [40].

2.6. Effect of Exercise on General Physical Functioning. Suppressed immune system function, poor cardiopulmonary fitness, reduced muscle strength, and fatigue may decrease the ability of a child with cancer or a childhood cancer survivor to participate comfortably in regular physical activity. Implementation of a program of exercise or EPA, on the other hand, may improve their strength and fitness and, if it alleviates fatigue, may increase ease of movement and enable activities that have a physical component. The evidence for efficacy of exercise and EPA programs to improve overall physical functioning and mobility in survivors of pediatric cancer is mixed. Among children with ALL, four different exercise intervention studies have documented the beneficial effects of a supervised training program or home-based exercise $[9,32]$ on general physical functioning, whereas three other studies have failed to find a positive effect of exercise on physical functioning [21, 33, 40]. Like the impact of exercise on fatigue, the impact of exercise on physical functioning appears to require that the intervention have a training effect. San Juan et al. in their series of three manuscripts $(n=7)$ demonstrated that a supervised training program among young children with ALL or in children following HCT, consisting of both resistance and aerobic exercises, improved not only muscle strength and cardiopulmonary fitness but also functional mobility as assessed by performance on three and ten meter timed up and go (TUG) tests (Cohen's $d-0.63$ to $-1.53, P<$ 0.05) [9]. Gohar et al. [32] reported improved gross motor function in nine children after implementing individualized home-based exercise programs during the early phases of treatment for ALL in nine children. However, the twelveweek supervised community-based intervention by Takken et al. [40] among 9 children during the maintenance phase of ALL treatment that had no training effect also had no impact on functional mobility. Additionally, Marchese et al. [21], who demonstrated improvements in ankle range of motion and knee extension strength, but no improvements in cardiopulmonary fitness $(P=0.25$, Cohen's $d=0.57)$ after implementation of a 16-week home program during maintenance therapy for children (intervention group $n=$ 13 , control group $n=15$ ) with ALL, also reported no improvements in performance on the TUG test $(P=0.17$, Cohen's $d=-0.55)$.

2.7. Effect of Exercise on Health-Related Quality of Life. Six of the studies we reviewed reported a health-related quality of life outcome (HRQOL) in response to exercise training or EPA [9, 21, 32, 34, 39]. Four reported a positive effect and two no effect. Positive effects were found in three studies with no control population, making it difficult to attribute the outcomes to the intervention rather than to developmental maturation or disease recovery. Gohar et al. [32] and Speyer et al. [39] both report overall improvement in HRQOL in response $(P<0.001$, Cohen's $d=1.43$ to 2.32$)$ to an individualized home-based exercise intervention [32] or to an in-hospital adapted physical activity intervention [39] among children during acute phases of treatment. Interestingly, the study by Gohar et al. [32] reported an initial reduction in HRQOL when chemotherapy was intensified during treatment among 9 children with ALL. San Juan et al. [9] also reported improved HRQOL $(P<0.05$, Cohen's $d=1.1$ ) in response to their 8 -week long supervised exercise intervention among 8 children following HCT, and Keats and Culos-Reed [34], in a group of 10 adolescent cancer survivors with mixed diagnoses, reported improved HRQOL ( $P=0.01$, Cohen's $d=0.34$ ) after a sixteen-week physical activity and educational intervention. These results in the adolescent survivors of mixed diagnoses persisted for at least one year following the end of the intervention. In contrast to the results of their study among children following HCT, San Juan et al. reported no effect of exercise training on HRQOL 
( $P=$ NS, Cohen's $d=0.31$ to 0.58 ) among 7 children who received 16-week supervised exercise intervention during maintenance therapy for ALL [9]. This finding is similar to that of Marchese et al., who also reported no differences between the intervention $(n=13)$ and control $(n=15)$ groups on HRQOL in their study of the effects of a 16week home-based PT intervention among children during maintenance therapy for childhood ALL [21].

\section{Conclusion}

It appears that exercise training can be safely undertaken during treatment for ALL and HCT with no major effects on the immune system and that exercise does not have a deleterious effect on growth factors during treatment for ALL. The published evidence is positive for the impact of exercise on muscle strength and flexibility and mixed for the impact of exercise intervention on cardiopulmonary fitness among children with ALL during maintenance therapy, among children following HCT, and among survivors exposed to cardiotoxic agents. Fatigue and general physical function are enhanced if the intervention generates a cardiopulmonary training effect. The evidence for the effects of exercise training on HRQOL in the childhood cancer population is mixed and difficult to disentangle from the effects of disease recovery and normal maturation. The early evidence suggests that supervised hospital training is effective, likely because compliance and training intensity are assured. Home- or community-based programs appear to be less effective. Unfortunately, supervised training is expensive and often unrealistic for families who may have to travel long distances to a center that specializes in cancer care.

Even though early results are promising, specific limitations in the existing literature do not allow us to yet be able to state with confidence that exercise interventions offer clear benefits during or after treatment for childhood cancer. There have only been four randomized trials, sample sizes have been small, and diagnosis groups included in the trials have been very limited (mostly ALL). Intent to treat type of analysis has not always been completed, and mechanisms to characterize the effects of participant dropout have not been employed. In addition, inconsistencies in exercise type, duration, and frequency, and outcome measurement prohibit conclusions that might guide how an individual clinician might prescribe exercise in practice.

Further research is needed. Studies designed to identify and characterize the type and intensity of exercise necessary to achieve clinically meaningful positive cardiopulmonary, musculoskeletal, symptom limiting, physical function, and quality of life outcomes in children with a variety of diagnoses are necessary. These interventions must be not only safe but also realistic and portable so that children, families, and long-term survivors can adopt and incorporate exercise and physical activity into their everyday lives when they are not near the specialized center that provides care for children with cancer. Additionally, larger well-designed randomized studies that employ strong statistical methodology and that evaluate the effects of participant dropout on the outcomes are important to see if the early results from these multiple small, mostly observational trials remain positive in larger populations of children with varied cancer diagnoses.

\section{References}

[1] M. A. Smith, N. L. Seibel, S. F. Altekruse et al., "Outcomes for children and adolescents with cancer: challenges for the twenty-first century," Journal of Clinical Oncology, vol. 28, no. 15, pp. 2625-2634, 2010.

[2] T. Hampton, "Cancer survivors need better care: new report makes recommendations," Journal of the American Medical Association, vol. 294, no. 23, pp. 2959-2960, 2005.

[3] S. B. J. Bhatia and A. T. Meadows, "Late effects of childhood cancer and its treatment," in Principles and Practice of Pediatric Oncology, P. D. Pizzo Pa, Ed., pp. 1490-1514, Lippincott Williams \& Wilkins, Philadelphia, Pa, USA, 2006.

[4] M. M. Hudson, A. C. Mertens, Y. Yasui et al., "Health status of adult long-term survivors of childhood cancer: a report from the childhood cancer survivor study," Journal of the American Medical Association, vol. 290, no. 12, pp. 1583-1592, 2003.

[5] A. C. Mertens, Y. Yasui, J. P. Neglia et al., "Late mortality experience in five-year survivors of childhood and adolescent cancer: the childhood cancer survivor study," Journal of Clinical Oncology, vol. 19, no. 13, pp. 3163-3172, 2001.

[6] K. C. Oeffinger, A. C. Mertens, C. A. Sklar et al., "Chronic health conditions in adult survivors of childhood cancer," New England Journal of Medicine, vol. 355, no. 15, pp. 1572-1582, 2006.

[7] S. Aznar, A. L. Webster, A. F. San Juan et al., "Physical activity during treatment in children with leukemia: a pilot study," Applied Physiology, Nutrition and Metabolism, vol. 31, no. 4, pp. 407-413, 2006.

[8] M. E. M. Jenney, E. B. Faragher, P. H. M. Jones, and A. Woodcock, "Lung function and exercise capacity in survivors of childhood leukaemia," Medical and Pediatric Oncology, vol. 24, no. 4, pp. 222-230, 1995.

[9] A. F. San Juan, C. Chamorro-Viña, J. L. Maté-Muñoz et al., "Functional capacity of children with leukemia," International Journal of Sports Medicine, vol. 29, no. 2, pp. 163-167, 2008.

[10] M. van Brussel, T. Takken, A. Lucia, J. van der Net, and P. J. M. Helders, "Is physical fitness decreased in survivors of childhood leukemia? A systematic review," Leukemia, vol. 19, no. 1, pp. 13-17, 2005.

[11] M. van Brussel, T. Takken, J. Van Der Net et al., "Physical function and fitness in long-term survivors of childhood leukaemia," Pediatric Rehabilitation, no. 3, pp. 267-274, 2006.

[12] J. T. Warner, W. Bell, D. K. H. Webb, and J. W. Gregory, "Daily energy expenditure and physical activity in survivors of childhood malignancy," Pediatric Research, vol. 43, no. 5, pp. 607-613, 1998.

[13] M. J. Adams, P. H. Hardenbergh, L. S. Constine, and S. E. Lipshultz, "Radiation-associated cardiovascular disease," Critical Reviews in Oncology/Hematology, vol. 45, no. 1, pp. 55-75, 2003.

[14] J. M. Argilés, F. J. López-Soriano, and S. Busquets, "Mechanisms to explain wasting of muscle and fat in cancer cachexia," Current Opinion in Supportive and Palliative Care, vol. 1, no. 4, pp. 293-298, 2007.

[15] K. S. Courneya, "Exercise in cancer survivors: an overview of research," Medicine and Science in Sports and Exercise, vol. 35, no. 11, pp. 1846-1852, 2003. 
[16] C. Duggan, L. Bechard, K. Donovan et al., "Changes in resting energy expenditure among children undergoing allogeneic stem cell transplantation," American Journal of Clinical Nutrition, vol. 78, no. 1, pp. 104-109, 2003.

[17] L. C. M. Kremer, E. C. Van Dalen, M. Offringa, J. Ottenkamp, and P. A. Voûte, "Anthracycline-induced clinical heart failure in a cohort of 607 children: long-term follow-up study," Journal of Clinical Oncology, vol. 19, no. 1, pp. 191-196, 2001.

[18] S. E. Lipshultz, "Exposure to anthracyclines during childhood causes cardiac injury," Seminars in Oncology, vol. 33, no. 3, supplement 8, pp. S8-S14, 2006.

[19] S. E. Lipshultz, S. R. Lipsitz, S. E. Sallan et al., "Chronic progressive cardiac dysfunction years after doxorubicin therapy for childhood acute lymphoblastic leukemia," Journal of Clinical Oncology, vol. 23, no. 12, pp. 2629-2636, 2005.

[20] R. D. K. S. Liu, M. J. M. Chinapaw, P. C. Huijgens, and W. V. Mechelen, "Physical exercise interventions in haematological cancer patients, feasible to conduct but effectiveness to be established: a systematic literature review," Cancer Treatment Reviews, vol. 35, no. 2, pp. 185-192, 2009.

[21] V. G. Marchese, L. A. Chiarello, and B. J. Lange, "Effects of physical therapy intervention for children with acute lymphoblastic leukemia," Pediatric Blood and Cancer, vol. 42, no. 2, pp. 127-133, 2004.

[22] A. F. San Juan, C. Chamorro-Vina, S. Moral et al., "Benefits of intrahospital exercise training after pediatric bone marrow transplantation," International Journal of Sports Medicine, vol. 29, no. 5, pp. 439-446, 2008.

[23] A. F. San Juan, S. J. Fleck, C. Chamorro-Viña et al., "Effects of an intrahospital exercise program intervention for children with leukemia," Medicine and Science in Sports and Exercise, vol. 39, no. 1, pp. 13-21, 2007.

[24] A. F. S. Juan, K. Wolin, and A. Lucía, "Physical activity and pediatric cancer survivorship," Recent Results in Cancer Research, vol. 186, pp. 319-347, 2011.

[25] I. M. Jóhannsdóttir, M. J. Hjermstad, T. Moum et al., "Increased prevalence of chronic fatigue among survivors of childhood cancers: A population-based study," Pediatric Blood and Cancer. In press.

[26] K. A. Meeske, S. K. Patel, S. N. Palmer, M. B. Nelson, and A. M. Parow, "Factors associated with health-related quality of life in pediatric cancer survivors," Pediatric Blood and Cancer, vol. 49, no. 3, pp. 298-305, 2007.

[27] C. L. Cox, M. Montgomery, K. C. Oeffinger et al., "Promoting physical activity in childhood cancer survivors," Cancer, vol. 115, no. 3, pp. 642-654, 2009.

[28] K. Y. Wolin, J. R. Ruiz, H. Tuchman, and A. Lucia, "Exercise in adult and pediatric hematological cancer survivors: an intervention review," Leukemia, vol. 24, no. 6, pp. 1113-1120, 2010.

[29] J. Cohen, Statistical Power Analysis for the Behavioral Sciences, Lawrence Erlbaum Associates, Hillsdale, NJ, USA, 1988.

[30] R. Blaauwbroek, M. J. Bouma, W. Tuinier et al., "The effect of exercise counselling with feedback from a pedometer on fatigue in adult survivors of childhood cancer: a pilot study," Supportive Care in Cancer, pp. 1-8, 2008.

[31] C. Chamorro-Vina, J. R. Ruiz, E. Santana-Sosa et al., "Exercise during hematopoietic stem cell transplant hospitalization in children," Medicine and Science in Sports and Exercise, vol. 42, no. 6, pp. 1045-1053, 2010.

[32] S. F. Gohar, M. Comito, J. Price, and V. Marchese, "Feasibility and parent satisfaction of a physical therapy intervention program for children with acute lymphoblastic leukemia in the first 6 months of medical treatment," Pediatric Blood and Cancer, vol. 56, no. 5, pp. 799-804, 2011.

[33] A. Hartman, M. L. Te Winkel, R. D. Van Beek et al., "A randomized trial investigating an exercise program to prevent reduction of bone mineral density and impairment of motor performance during treatment for childhood acute lymphoblastic leukemia," Pediatric Blood and Cancer, vol. 53, no. 1, pp. 64-71, 2009.

[34] M. R. Keats and S. N. Culos-Reed, "A community-based physical activity program for adolescents with cancer (project TREK): program feasibility and preliminary findings," Journal of Pediatric Hematology/Oncology, vol. 30, no. 4, pp. 272-280, 2008.

[35] A. B. Ladha, K. S. Courneya, G. J. Bell, C. J. Field, and P. Grundy, "Effects of acute exercise on neutrophils in pediatric acute lymphoblastic leukemia survivors: a pilot study," Journal of Pediatric Hematology/Oncology, vol. 28, no. 10, pp. 671-677, 2006.

[36] L. J. Moyer-Mileur, L. Ransdell, and C. S. Bruggers, "Fitness of children with standard-risk acute lymphoblastic leukemia during maintenance therapy: response to a home-based exercise and nutrition program," Journal of Pediatric Hematology/Oncology, vol. 31, no. 4, pp. 259-266, 2009.

[37] J. R. Ruiz, S. J. Fleck, J. L. Vingren et al., "Preliminary findings of a 4-month intrahospital exercise training intervention on IGFs and IGFBPs in children with leukemia," Journal of Strength and Conditioning Research, vol. 24, no. 5, pp. 12921297, 2010.

[38] A. M. Sharkey, A. B. Carey, C. T. Heise, and G. Barber, "Cardiac rehabilitation after cancer therapy in children and young adults," American Journal of Cardiology, vol. 71, no. 16, pp. 1488-1490, 1993.

[39] E. Speyer, A. Herbinet, A. Vuillemin, S. Briançon, and P. Chastagner, "Effect of adapted physical activity sessions in the hospital on health-related quality of life for children with cancer: a cross-over randomized trial," Pediatric Blood and Cancer, vol. 55, no. 6, pp. 1160-1166, 2010.

[40] T. Takken, P. van der Torre, M. Zwerink et al., "Development, feasibility and efficacy of a community-based exercise training program in pediatric cancer survivors," Psycho-Oncology, vol. 18, no. 4, pp. 440-448, 2009.

[41] C. H. Yeh, J. P. Man Wai, U. S. Lin, and Y. C. Chiang, "A pilot study to examine the feasibility and effects of a home-based aerobic program on reducing fatigue in children with acute lymphoblastic leukemia," Cancer Nursing, vol. 34, no. 1, pp. 3-12, 2011.

[42] P. S. Hinds, M. Hockenberry, S. N. Rai et al., "Clinical Field Testing of an Enhanced-Activity Intervention in Hospitalized Children with Cancer," Journal of Pain and Symptom Management, vol. 33, no. 6, pp. 686-697, 2007.

[43] A. F. San Juan, S. J. Fleck, C. Chamorro-Viña et al., "Earlyphase adaptations to intrahospital training in strength and functional mobility of children with leukemia," Journal of Strength and Conditioning Research, vol. 21, no. 1, pp. 173-177, 2007.

[44] American College of Sports Medicine, ACSM's Guidelines for Exercise Testing and Prescription, Williams \& Wilkins, Philadelphia, Pa, USA, 2000.

[45] E. Golden, B. Beach, and C. Hastings, "The pediatrician and medical care of the child with cancer," Pediatric Clinics of North America, vol. 49, no. 6, pp. 1319-1338, 2002.

[46] C. S. M. Oude Nijhuis, S. M. G. J. Daenen, E. Vellenga et al., "Fever and neutropenia in cancer patients: the diagnostic role 
of cytokines in risk assessment strategies," Critical Reviews in Oncology/Hematology, vol. 44, no. 2, pp. 163-174, 2002.

[47] J. Skinner, J. L. Finlay, P. M. Sondel, and M. E. Trigg, "Infectious complications in pediatric patients undergoing transplantation with T lymphocyte-depleted bone marrow," Pediatric Infectious Disease, vol. 5, no. 3, pp. 319-324, 1986.

[48] A. S. Fairey, K. S. Courneya, C. J. Field, and J. R. Mackey, "Physical exercise and immune system function in cancer survivors: a comprehensive review and future directions," Cancer, vol. 94, no. 2, pp. 539-551, 2002.

[49] D. M. Chun, C. B. Corbin, and R. P. Pangrazi, "Validation of criterion-referenced standards for the mile run and progressive aerobic cardiovascular endurance tests," Research Quarterly for Exercise and Sport, vol. 71, no. 2, pp. 125-134, 2000.

[50] L. H. Gerber, K. Hoffman, U. Chaudhry et al., "Functional outcomes and life satisfaction in long-term survivors of pediatric sarcomas," Archives of Physical Medicine and Rehabilitation, vol. 87, no. 12, pp. 1611-1617, 2006.

[51] V. G. Marchese, L. A. Chiarello, and B. J. Lange, "Strength and functional mobility in children with Acute Lymphoblastic Leukemia," Medical and Pediatric Oncology, vol. 40, no. 4, pp. 230-232, 2003.

[52] K. K. Ness, E. B. Morris, V. G. Nolan et al., "Physical performance limitations among adult survivors of childhood brain tumors," Cancer, vol. 116, no. 12, pp. 3034-3044, 2010.

[53] N. R. Clanton, J. L. Klosky, C. Li et al., "Fatigue, vitality, sleep, and neurocognitive functioning in adult survivors of childhood cancer: a report from the childhood cancer survivor study," Cancer, vol. 117, no. 11, pp. 2559-2568, 2011.

[54] M. J. Hockenberry, M. C. Hooke, M. Gregurich, K. McCarthy, G. Sambuco, and K. Krull, "Symptom clusters in children and adolescents receiving cisplatin, doxorubicin, or ifosfamide," Oncology Nursing Forum, vol. 37, no. 1, pp. E16-27, 2010. 


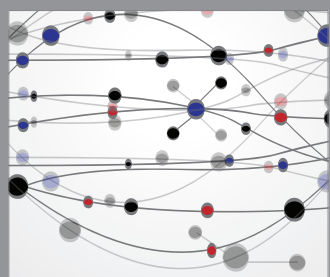

The Scientific World Journal
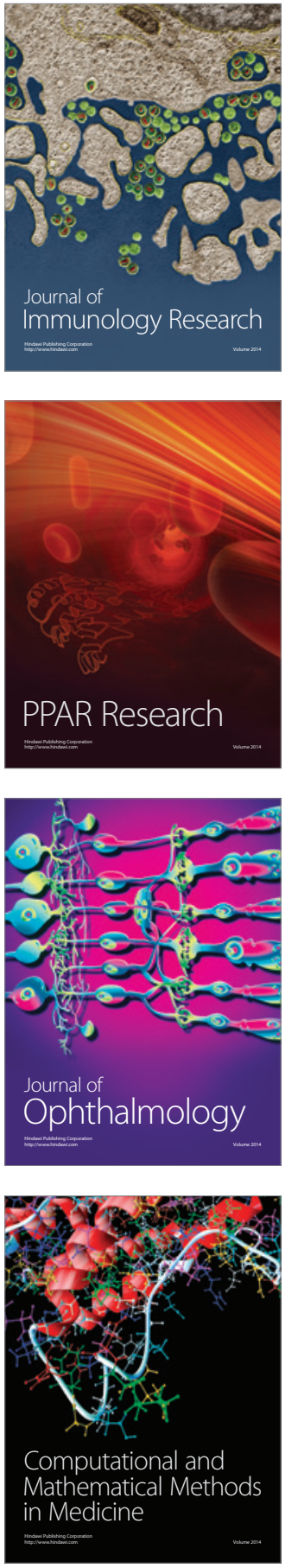

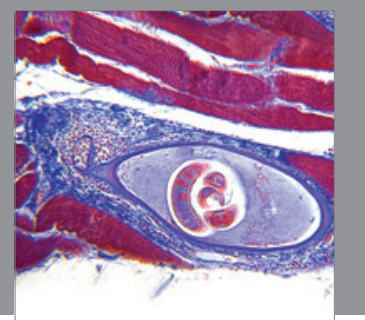

Gastroenterology

Research and Practice
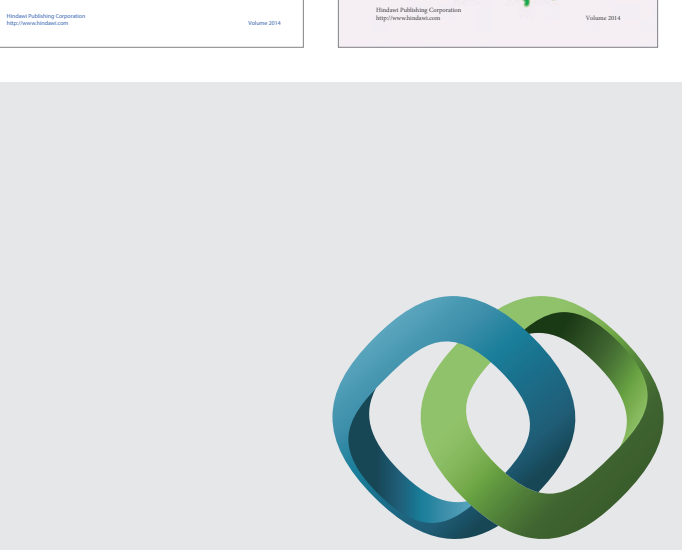

\section{Hindawi}

Submit your manuscripts at

http://www.hindawi.com
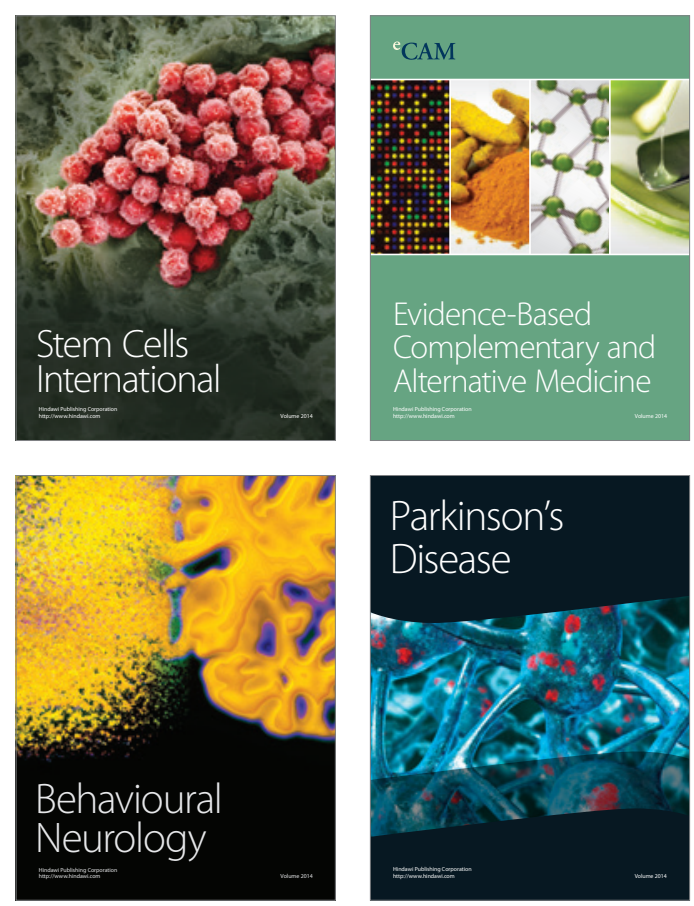

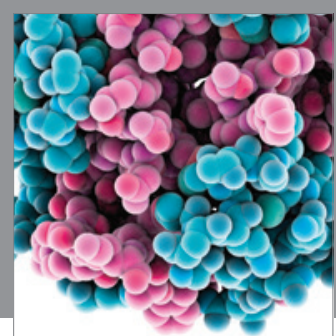

Journal of
Diabetes Research

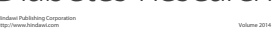

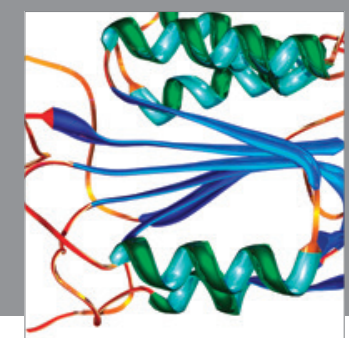

Disease Markers
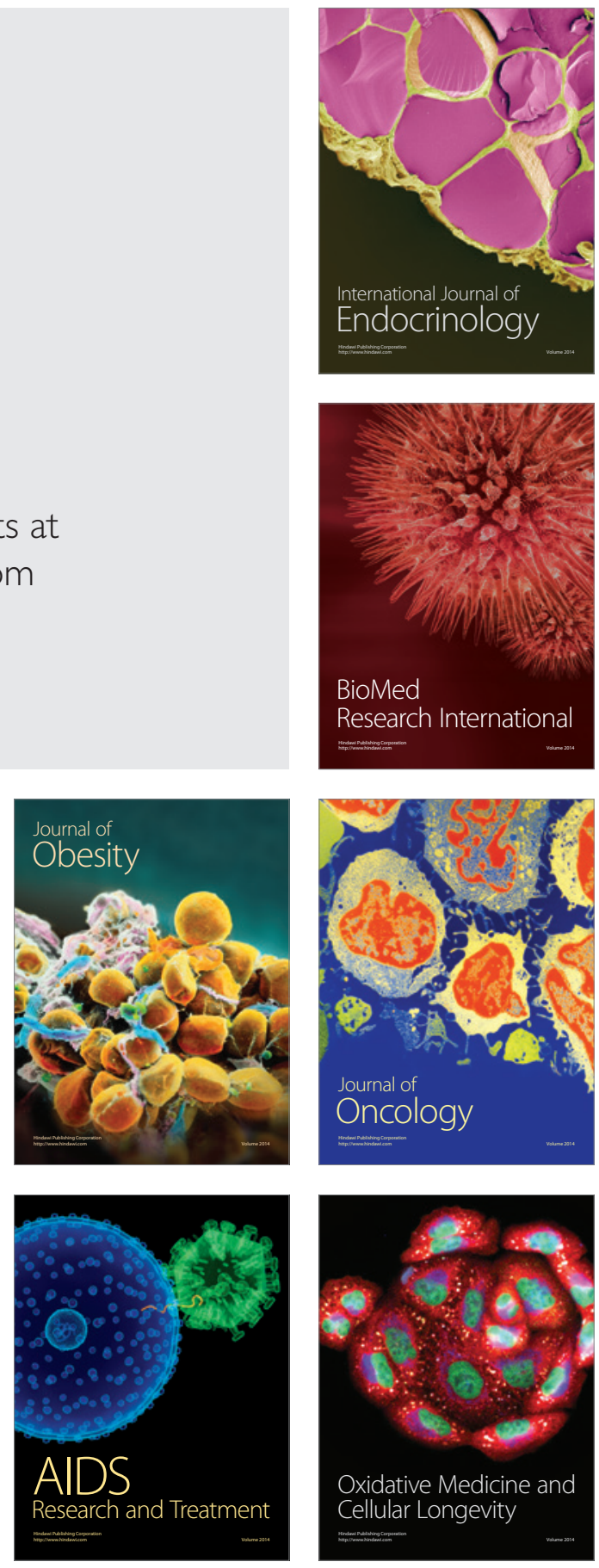